\title{
Persistent effect of noise on the acoustic startle reflex in the rat
}

\author{
ROBERT N. CORY and JAMES R. ISON \\ University of Rochester, Rochester, New York 14627
}

\begin{abstract}
Continuous noise facilitates acoustic startle reflexes in the rat. Rats were exposed to noise for $23 \mathrm{~h}$ (Experiment 1) or to $23 \mathrm{~h}$ of startle eliciting stimuli at the rate of $1 / \mathrm{min}$ (Experiment 2). Facilitation was reduced following habituation in Experiment 2, but was unaffected by prolonged noise exposure in Experiment 1. Reflex inhibition produced by a brief noise was not altered by habituation. Prior experiments show that increases in intensity of continuous noise engage two disparate processes which affect the acoustic startle reflex, one facilitatory (arousal) and one inhibitory (masking). The present data reveal that arousal is not diminished by prolonged noise exposure. The loss of facilitation following reflex habituation may be attributed to its increased susceptibility to masking, or to a direct effect of stimulus repetition on the arousal process normally associated with the noise background.
\end{abstract}

The strength of the rat's acoustic startle reflex is affected by variation in the homogeneity and intensity of the acoustic background present at the time of the eliciting stimulus. A steady noise of moderate intensity facilitates the reflex, whereas a punctate noise which immediately precedes the eliciting stimulus inhibits it. The two experiments reported here examined the persistence of these modulating influences following prolonged continuous exposure to the noise and prolonged repetitive exposure to the eliciting stimulus.

\section{EXPERIMENT 1}

The facilitative effect of continuous noise on the rat's acoustic startle reflex is apparent within $100 \mathrm{msec}$ of noise onset and becomes asymptotic within 2,000 msec thereafter (Hoffman \& Wible, 1969). It has been concluded that this source of reflex potentiation does not decay when stimulation is maintained beyond 2,000 msec (Davis, 1974). This conclusion follows from an experimental result provided by Hoffman, Marsh, and Stein (1969), who used two background conditions, either pulsed or continuous noise, and found that the advantage of the steady noise was stable over a 6-h exposure period. However, the outcome of this procedure is difficult to interpret because pulsed noise against a silent background inhibits the startle reflex. In the absence of a silent neutral baseline, the experiment provided no conclusive evidence for facilitation: the difference between the two conditions may have been due to inhibition

Reprint requests should be directed to Professor James R. Ison, Department of Psychology, University of Rochester, Rochester, New York 14627. This research was supported by grants from the NINCDS, NS-12443, and from the NIEHS, ES-01247. rather than facilitation. Its stability over the 6-h period might characterize only the inhibitory process, which has been otherwise shown to be a stable phenomenon (Ison, Hammond, \& Krauter, 1973). The present experiment provided the appropriate silent background condition as a control and extended the period of exposure to $23 \mathrm{~h}$. The scheduling of tests about $24 \mathrm{~h}$ apart avoided effects on pre- and posttests that might occur from a different location within the circadian rhythm (Horlington, 1970; Sollberger \& Davis, 1972). The experiment provided a striking demonstration of the persistence of reflex facilitation over this prolonged period.

\section{Method}

Subjects. The animals were four female and four male albino rats, about 7 weeks old, derived from stock obtained from the Holtzman Co., Madison, Wisconsin. A reverse light/dark cycle was maintained from birth.

Apparatus. The experimental setup was housed in a singlewall Industrial Acoustics Co., audiometric testing room, approximately $2.4 \mathrm{~m}$ to a side. The animal was enclosed in a small chamber (approximately $6 \times 9 \times 18 \mathrm{~cm}$ ) with brass rod walls and a floor made of two steel plates separated by a midline gap of $1.2 \mathrm{~cm}$. The chamber was held rigidly in a frame by compression springs. Food and water were available in the experiment ad lib. A drinking tube connected to an overhead reservoir protruded through the top of the cage, and food pellets were lodged into the brass rods. An accelerometer (Statham, Model SA-2-300), mounted under the cage, detected the startle movement. The startle reaction was displayed on the paper output of a Beckman polygraph with simultaneous readout on a Fluke $8000 \mathrm{~A}$ digital multimeter which integrated activity in the 100 -msec epoch following an eliciting stimulus. The startle stimulus (a $10-\mathrm{kHz}$ tone of $20 \mathrm{msec}$ duration with a 5 -msec rise and decay time, at $110 \mathrm{~dB}$ re $20 \mu \mathrm{N} / \mathrm{m}^{2}$ ) was provided by a Hewlett Packard oscillator and gated through an electronic switch by electronic timers. The stimulus was amplified and delivered to a Calrad high-frequency tweeter following passage through a relay circuit. This circuit was controlled by the electronic switch and gated out all input to the speaker except during the "on" cycle of the switch. The background noise stimulus was provided by a Grason-Stadler white- 
noise generator delivered through a second electronic switch and similar gating circuit to two 4-in. $(10.2-\mathrm{cm})$ cone speakers. The white noise had a 5-msec rise and decay time and was set at $70 \mathrm{~dB}$ (re $20 \mu \mathrm{N} / \mathrm{m}^{2}$ ). In its absence the ambient background level in the chamber was $27 \mathrm{~dB}$ (re $20 \mu \mathrm{N} / \mathrm{m}^{2}$ ) for frequencies from approximately .1 to $10 \mathrm{kHz}$. Stimulus intensities were measured in the cage using a General Radio sound-level meter (1561) and impact noise analyzer (1556-B).

Procedure. The experimental procedure lasted approximately $24 \mathrm{~h}$. It began when the animal was placed in the test chamber and, 3 min later, was presented with 20 startle stimuli at an interstimulus interval of $15-20 \mathrm{sec}$. One-half of these stimuli were given in the 27-dB ambient noise level ( $\mathrm{S} 1$ alone) and one-half were given about $10 \mathrm{sec}$ after the onset of the $70-\mathrm{dB}$ noise (S1S2). One-half of the animals began the series with one sort of trial and one-half began it with the other, with a quasi-random order thereafter. The animals were left in the cage for $23 \mathrm{~h}$ and then received a second series of trials identical to their first series. During the $23 \mathrm{~h}$, the animals were either continuously exposed to the 70-dB noise background or left in the relatively silent $27-\mathrm{dB}$ ambient background. A similar pretest/treatment/ posttest sequence was run 2 weeks later with the order of noise treatment or silence counterbalanced across animals. Additionally, the type of first posttest trial was counterbalanced and was S1-S2 for half and S2-alone for half. As a result of this latter counterbalancing, half of the animals within each condition had their first startle stimulus preceded by a background change from 27 to $70 \mathrm{~dB}$ or the reverse. For the other half, the eliciting stimulus was presented in the treatment exposure condition with no preceding perturbation.

Testing was done at approximately $2100 \mathrm{~h}, 1 \mathrm{~h}$ before the onset of the 12-h light period. At this time, the room was dimly illuminated with a $25-\mathrm{W}$ red incandescent light bulb. In the light segment of the cycle, the room was illuminated with a $60-\mathrm{W}$ bulb. The animal was monitored during testing over a closedcircuit TV system, and was also irregularly watched during the 23-h treatment. In the analyses of the ensuing data, the total of each animal's integrated startle reactions (summed over all 80 trials) was set at 100 and its score on each individual trial was then expressed as a proportion $(\times 100)$ of that total. This normalizing procedure removes individual differences in overall reactivity so that each animal's relative performance in each condition is equally weighted in the analysis.

\section{Results and Discussion}

Figure 1 gives the mean relative startle amplitude on S2-alone and S1-S2 trials for the pretests (summed together) and for the posttests which followed either $23 \mathrm{~h}$ of $70 \mathrm{~dB}$ noise or $23 \mathrm{~h}$ of relative silence. Overall, the startle response was greater in the presence of the noise by a factor of about 2 to $1[F(1,6)=32.47$, $p<.01]$. Of particular interest for the purposes of this investigation is the failure to find any indication that the 23-h exposure to noise diminished its effectiveness. However, regardless of the type of exposure, the size of the reflex increased in the posttest $[F(1,6)=17.13, p<.01]$. There was no interaction between type of exposure and this posttest advantage $(F<1)$. Analyses across trials indicated that the startle response declined $[F(9,54)=6.07$, $\mathrm{p}<.01]$ with significant quadratic $(\mathrm{p}<.05)$ and cubic $(p<.01)$ trials. The decline did not interact with conditions. Thus, the major conclusion to be drawn from this experiment is that the noise maintains its

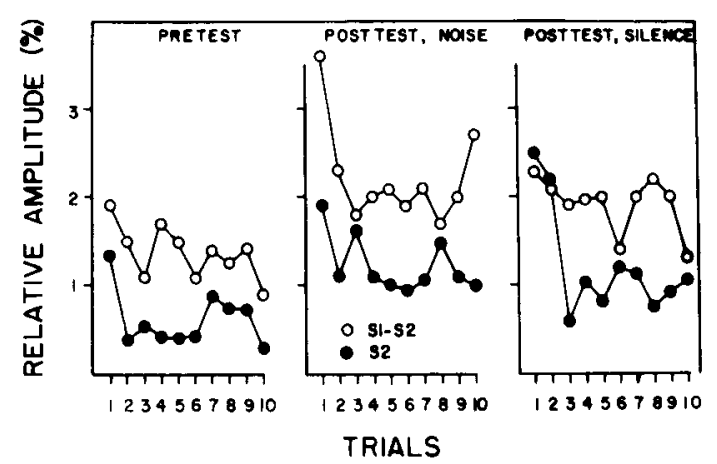

Figure 1. Relative startle amplitudes on control trials in silence (S2) or in the presence of noise (S1-S2) in pretest and on posttests, following 23-h exposure to noise or to silence.

effectiveness in facilitating the startle reflex even though the animal had been exposed to the noise for about $23 \mathrm{~h}$.

The data obtained on the first trials of the posttest are worthy of some additional attention, however, because they seem to suggest that the advantage normally obtained in the noise condition was temporarily absent if the animal had been just exposed to $23 \mathrm{~h}$ of silence. At the beginning of the posttest, it may be seen that following $23 \mathrm{~h}$ of silence the startle response elicited in that condition was just as large as that elicited in the presence of noise. This appears to result from something that has happened to silence rather than a decrement in the facilitative effect of noise, and we suspect that these data reveal another source of reflex invigoration. Prolonged innocuous exposure to noise or silence may lead to a state of relaxation or drowsiness, conducive to startle reflexes (Oswald, 1959). Davis (1974) has shown that if the rat is left undisturbed in the presence of noise, then the next following startle reflex is increased in vigor. He followed this effect out for $45 \mathrm{~min}$, and found that it reached asymptote at around $30 \mathrm{~min}$. The present data suggest that this sort of reflex potentiation may occur in silence also if the animal is left undisturbed for a sufficiently long period of time.

\section{EXPERIMENT 2}

Although exposure to noise alone is without apparent effect, there is some reason to suppose that repetitive elicitation of the acoustic startle reflex may reduce the facilitating effect of noise upon it. The experiment of Hoffman et al. (1969) had included animals which were exposed to brief trains of startle stimuli each hour, these being superimposed on the steady or on the pulsed noise. As testing continued, the vigor of the reflexes observed in these two noise conditions declined and the curves 
converged. The fact of convergence suggests that with reflex habituation there is either a decrement in the inhibiting effect of the pulsed noise or a loss of facilitation from the steady noise. Some support for the latter possibility is provided in an experiment by Davis (1974). Two groups of animals received 600 repetitions of an acoustic startle stimulus, one group in a $70-\mathrm{dB}$ background, the other in a $60-\mathrm{dB}$ background. The startle reflex in both groups showed a steady decline, but the decline was greater in the group with the more intense background, so that by the end of training the normally invigorating effect of that higher noise level had been eliminated.

Experiment 2 provided further evidence on the suggested relationship between reflex repetition and the reflex modulating consequences of background noise. We tested for both facilitating and inhibitory effects of steady and punctate noises, by contrasting the two noise manipulations with a control silent background. Reflex inhibition was unaffected by stimulus repetition, whereas reflex facilitation was much reduced.

\section{Method}

Subjects. The animals were seven naive male albino rats obtained from the Holtzman Co., Madison, Wisconsin, and run at a weight of $200-250 \mathrm{~g}$. Maintenance conditions were the same as in the preceding experiment.

Apparatus. The apparatus was identical to that used previously except that the response amplitudes were recorded on a Grass polygraph and measured as millimeters' pen deflection.

Procedure. The animal was placed in the test chamber $3 \mathrm{~min}$ prior to the pretest. Thirty tones $(10 \mathrm{kHz}, 110 \mathrm{~dB}, 20 \mathrm{msec}$ duration) were presented, 10 in each of three conditions: (1) S2-alone, given in the ambient $27-\mathrm{dB}$ background to establish the control level: (2) S1-S2 $10 \mathrm{sec}$, in which S2 was given $10 \mathrm{sec}$ after the onset of S1, an $11-\mathrm{sec}, 70-\mathrm{dB}$ white noise (5-msec rise and decay time): (3) $\mathrm{S} 1-\mathrm{S} 260 \mathrm{msec}$, in which $\mathrm{S} 2$ was presented $60 \mathrm{msec}$ after the onset of $\mathrm{S} 1$, a $20 \mathrm{msec}, 70-\mathrm{dB}$ white noise prepulse $(5-\mathrm{msec}$ rise and decay time). The intertrial interval averaged $20 \mathrm{sec}$. These conditions were randomly presented by arranging them in 6 by 5 matrices so that each condition occurred twice in each of the five rows. Over the next $23 \mathrm{~h}$ (which varied with a range of $20 \mathrm{~min}$ ), the caged animals were kept in the 27-dB background and given S2 once every minute (for a total of 1,380-1,400 stimuli). At the end of this time, a posttest identical to the pretest was given. In the treatment of the ensuing pre- and posttest data, the measurements for each animal were summed and the totals for each of the three conditions in each test were then expressed as a proportion of the animal's grand total.

\section{Results and Discussion}

Figure 2 shows mean startle amplitudes in the pretest and posttest for the S2-alone condition, the S1S2 10-sec condition, and the S1-S2 60-msec condition. In the pretest, as is usual, the startle reflex was larger in the presence of the stable white noise (by an average of $279 \%$ ) and smaller following the punctate noise at the brief interstimulus interval (by an average of $42 \%$ ). In the posttest, the size of the S1alone control response was diminished by about $25 \%$. Although the inhibiting effectiveness of the brief

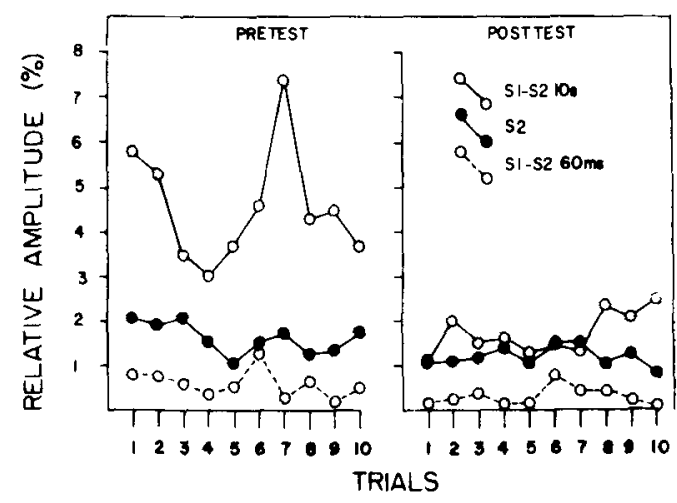

Figure 2. Relative startle amplitudes on control trials in silence (S2), on trials briefly preceded by noise onset (S1-S2 $60 \mathrm{msec})$, and in the presence of noise ( $\mathrm{S} 1-\mathrm{S} 210 \mathrm{sec})$, before (pretest) and after (posttest) habituation of the startle reflex.

stimulus was maintained (at $48 \%$ ), the facilitating influence of the larger noise was much reduced (to an average of but $43 \%$ facilitation). Analysis of variance of the mean startle amplitudes showed a reliable decline from pre- to posttest $[F(1,6)=6.94$, $p<.05]$, a reliable effect of conditions $[F(2,12)=$ $20.15, p<.01$ l, and a reliable interaction of conditions and test $[\mathrm{F}(2,12)=9.12, \mathrm{p}<.01]$. Analysis of the changes in facilitation between the tests showed that its decrement was reliable $[\mathrm{t}(6)=2.83$, $\mathrm{p}<.05]$, whereas inhibition was not altered $(t=.22)$.

Although there was an overall decrement in the S2alone condition from pretest to posttest, it was variously manifested in seven animals. One actually showed a reliable increase in reflex amplitudes on the 2nd day, but even this animal showed a substantial loss in noise-produced facilitation. Within the group of animals, there was no apparent relationship between the amount of habituation of the control reflex and the decrement in the effect of the noise.

The loss of response strength obtained in the S2alone control reflex was surprisingly small given the large number of intervening habituation trials. This may have resulted in part because of the change in context between "habituation training" and "habituation test"; the reduction in the temporal interval between successive tones and the addition of the additional noise stimuli in the posttest could be expected to have had a dishabituating effect. In addition, we noticed that there were major shifts in reflex strength over the course of the $23 \mathrm{~h}$. Some of these were of considerable duration. Figure 3 shows five separate 60 -min periods for the first animal with reflex amplitudes plotted cumulatively within each hour. Cumulative plots tend to obscure local variations in reflex strength and provide a picture of relatively stable long-term influences. It may be seen that the rate of response accumulation in the first hour was considerably greater than that seen in the final hour, by a factor of about 3 to 1 . This is the 


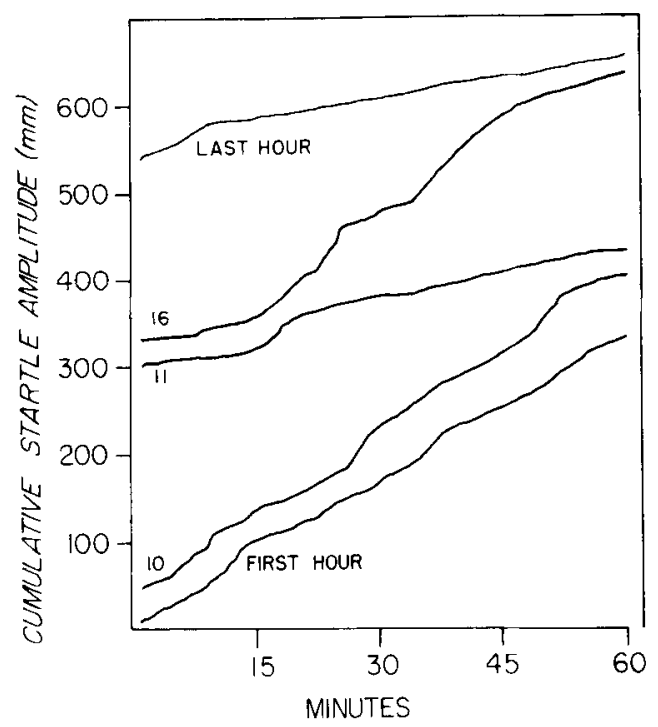

Figure 3. Cumulative response amplitudes for Subject 1 in five 60-min periods taken from 23 consecutive hours in which the subject received a startle-eliciting stimulus every $60 \mathrm{sec}$. The hours depicted are the 1st, the 10th, the 11th, the 16th, and the last.

expected effect of repetition of the eliciting stimulus, but the rate of growth did not steadily diminish in the intervening hours, but, rather, showed a number of both increases and decreases.

The other data in Figure 3 show cumulative responses in the hours about half (10 and 11) and two-thirds (16) into the session. For much of the 10th hour, the average response was about equal to that obtained in the 1 st hour. Then, after three relatively large responses, the animal settled into a 20-min period of small responses of the same size as those later seen throughout the $23 \mathrm{rd}$ hour. These small responses were maintained to the end of the 11 th hour after one intervening burst of large responses lasting for $8 \mathrm{~min}$. The 16th hour is interesting because at its beginning and end the responses were just as small as those observed in the final hour, but in the central $30 \mathrm{~min}$ there were two 10 min periods of vigorous responding.

It will be remembered that food and water were available to these animals, and although the cage was small they could engage in a diverse variety of behaviors, e.g., grooming, sleeping, eating, drinking, etc. We have previously reported (Ison \& Krauter, 1975) that the startle reflex is larger when the rat drinks, and we have observed on numerous occasions that the reflex tends to be smaller if the animal is grooming or is otherwise active at the time of the startle stimulus. The startle stimuli must have been superimposed on these behaviors from time to time, and reflex amplitudes could then be expected to vary accordingly. But such influences may be expected to be transitory and thus exert only local variation in reflex vigor. In contrast, examination of the 23-h records revealed a number of homogeneous runs of substantial length of the sort displayed in Figure 3. These runs probably reflect periods of stable reflex excitability associated with particular states of arousal in, e.g., sleep, etc., which may be imposed on the diurnal rhythm of the startle reaction reported by Sollberger and Davis (1972). Unfortunately, we were not equipped to examine the behavioral and state correlates of the long-term homogeneous runs that this procedure yields, but we suspect that such study would yield fruitful insights into the determinants of the startle reflex.

To return to the focus of this second experiment, although the loss of facilitation following reflex habituation is empirically established, its understanding remains unclear. It is commonly understood that a steady noise yields arousal, and that its facilitation of the startle reflex is part of a widespread process which affects a variety of both reflexive and nonreflexive behaviors. It may be that the animal's exposure to the many startle stimuli directly reduces the ability of the noise background to evoke arousal. It may be presumed that acoustic startle stimuli also evoke the arousal reaction, and that its repetitive evocation in this context leads to its habituation to both the startle stimulus and, via generalization, to white noise as well. The puzzle for this account is why the arousing consequence of noise might be habituated by repetitive startle stimuli, yet be unaffected by its chronic elicitation in Experiment 1.

There is a second possibility, raised by Davis (1974). Background noise has two effects on acoustic startle behavior: at low-to-moderate noise levels, the reflex is greater in the presence of a more intense noise, but at moderate-to-intense noise levels this relationship is reversed. A nonmonotone function, the "inverted-U," relates acoustic startle vigor to background noise intensity (Ison \& Hammond, 1971). A second explanation of the relationship between the invigorating effects of noise and stimulus repetition is that the habituated response is more sensitive to the processes responsible for the terminal limb of the inverted $U$. The declining portion of the function has been attributed in part to a sensory masking of the eliciting stimulus by the intense background noise (Ison \& Hammond, 1971). Davis (1974) has suggested that habituation may reduce the functional intensity of the eliciting stimulus. This might then result in its being more readily masked by the noise, which would then tend to counteract the positive arousing effect.

\section{REFERENCES}

Davis, M. Signal-to-noise ratio as a predictor of startle amplitude and habituation in the rat. Journal of Comparative and Physiological Psychology, 1974, 86, 812-825. 
Hoffman, H. S., Marsh, R. R., \& Stein, N. Persistence of background acoustic stimulation in controlling startle. Journal of Comparative and Physiological Psychology, 1969, 68, 280-283.

Hoffman, H. S., \& Wible, B. L. Temporal parameters in startle facilitation by steady background signals. Journal of the Acoustical Society of America, 1969, 45, 7-12.

Horlington, M. Startle response circadian rhythm in rats: Lack of correlation with motor activity. Physiology \& Behavior, 1970, $5,49-53$.

Ison, J. R., \& Hammond, G. R. Modification of the startle reflex in the rat by changes in the auditory and visual environments. Journal of Comparative and Physiological Psychology, 1971, $73,435-452$.

Ison, J. R., Hammond, G. R., \& Krauter, E. E. Effects of experience on stimulus produced reflex inhibition in the rat.
Journal of Comparative and Physiological Psychology, 1973, 83, 324-336.

Ison, J. R., \& Krauter, E. E. Acoustic startle reflexes in the rat during consummatory behavior. Journal of Comparative and Physiological Psychology, 1975, 89, 39-49.

OswaLd, I. Sudden bodily jerks on falling asleep. Brain, 1959, 82, 92-103.

Sollberger, A., \& Davis, M. Diurnal rhythm of the startle response: A statistical analysis. Journal of Interdisciplinary Cycle Research, 1972, 3, 313-325.

(Received for publication July 31, 1978; revision accepted December $27,1978$. ) 